\title{
Dynamics of metallic stripes in cuprates
}

\author{
J. Lorenzana ${ }^{1}$ and G. Seibold ${ }^{2}$ \\ ${ }^{1}$ CONICET, Centro Atómico Bariloche, 8400 S. C. de Bariloche, \\ Argentina and INFM Center for Statistical Mechanics and Complexity, \\ Università di Roma "La Sapienza", P. Aldo Moro 2, 00185 Roma, Italy \\ ${ }^{2}$ Institut für Physik, BTU Cottbus, PBox 101344, 03013 Cottbus, Germany
}

(Dated: November 2, 2018)

\begin{abstract}
We study the dynamics of metallic vertical stripes in cuprates within the three-band Hubbard model based on a recently developed time-dependent Gutzwiller approximation. As doping increases the optical conductivity shows transfer of spectral weight from the charge transfer band towards i) an incoherent band centered at $1.3 \mathrm{eV}$, ii) a Drude peak, mainly due to motion along the stripe, iii) a low-energy collective mode which softens with doping and merges with ii) at optimum doping in good agreement with experiment. The softening is related to the quasidegeneracy between $\mathrm{Cu}$ centered and $\mathrm{O}$ centered mean-field stripe solutions close to optimal doping.
\end{abstract}

PACS numbers: 71.45.Lr, 71.10.Hf, 74.72.-h, 78.30.Er

The doping-dependent evolution from insulating behavior to a strange metal in the superconducting cuprates emerges dramatically in the normal state optical conductivity. Slightly doped cuprates show a small or no Drude peak and doping-induced transfer of spectral weight from the charge-transfer $(\sim 2 \mathrm{eV})$ to a mid-IR $(\mathrm{MIR})$ band at $\sim 0.5 \mathrm{eV}[1$, 2]. Upon further doping the system progressively metallizes as is evident from the prominent Drudelike peak that develops at zero energy. A remarkable effect of doping is that the MIR band strongly softens and merges with the Drude peak, resulting in a feature that cannot be fitted by a conventional Drude model. A variety of alternative theories [3, [- [] have been proposed in order to describe this feature. Clearly the identification of this low-energy MIR (LEMIR) band is of paramount importance to understand the physics of these materials. The low-doping behavior has been explained in terms of the random-phase-approximation (RPA) electronic excitations of single-hole Hartree-Fock states in $\mathrm{CuO}_{2}$ layers [0 softening of the LEMIR band) could not be explained due to difficulties with the HF ground state.

The softening of the LEMIR band in $\mathrm{La}_{2-x} \mathrm{Sr}_{x} \mathrm{CuO}_{4}$ (LSCO) is accompanied by the appearance of another (much less discussed) band at $1.3 \mathrm{eV}$ 11, 2, 6. 6. This high energy MIR (HEMIR) band is well pronounced in optical absorption through LSCO thin films [2], and electron energy loss spectroscopy 6 , where it develops as a function of doping. Moreover LEMIR and HEMIR are also detected in photodoped experiments on LSCO[7. The HEMIR has not been clearly resolved by reflectivity in $\mathrm{YBa}_{2} \mathrm{Cu}_{3} \mathrm{O}_{6+\delta}(\mathrm{YBCO})$, but a strong broad feature at the right energy appears in photodoped transmission experiments [8]. As far as we know no microscopic explanation of the HEMIR exists so far.

Another important aspect of layered cuprates that has emerged in the last years is the rearrangement of doped holes in antiferromagnetic (AF) domain walls 9, 10, 11,

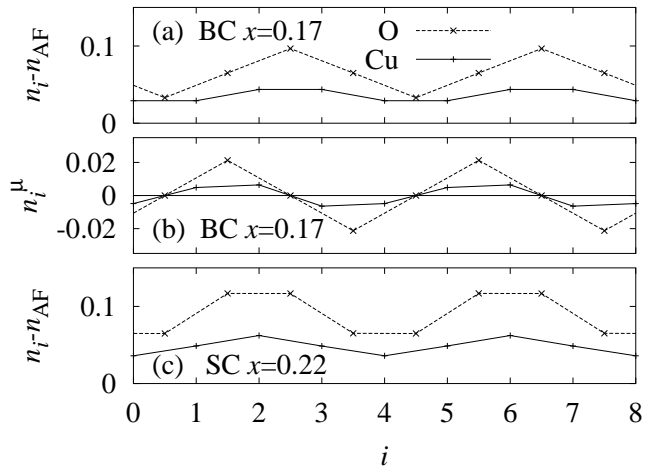

FIG. 1: Charge density minus undoped charge density as a function of atomic position in the direction perpendicular to the $d=4$ stripe for the BC solution at $x=0.17$ (a) and the SC solution at $x=0.22(\mathrm{c})$. (b) shows the transition charge for the LEMIR collective mode of the panel (a) solution.

12, 13, 14. These one-dimensional (1D) structures called stripes were predicted by mean-field theories 15 .

Recently we have presented a computation of metallic mean-field stripes 16] within an unrestricted Gutzwiller approximation 17 (GA). The behavior of the magnetic incommensurability $\epsilon=1 /(2 d)[11,12,13,14$, $(d$ is the distance between charged stripes in units of the lattice constant), chemical potential 18, 19], and transport experiments 20, 21 as a function of doping have been explained in a parameter free way 16 .

In this work we investigate the optical conductivity in terms of linear excitations around the metallic meanfield stripes of Ref. [16]. RPA fluctuations are added through a recently developed time-dependent GA labeled GA+RPA 22]. The stripes show a strong Drude component in the stripe direction, of magnitude similar to the experimental one. In addition the single-hole[] MIR band splits into two bands in good accord with the HEMIR and LEMIR (Fig. 3). The HEMIR band can be 
understood in terms of incoherent transitions between band states of a stripe at mean-field level (Fig. 2). The LEMIR band is a collective mode associated with soft lateral displacements of the stripe. As doping increases the collective mode shifts to lower energy and merges with the Drude part, providing an explanation for the softening of the LEMIR band in cuprates. This softening is due to the quasidegeneracy between $\mathrm{O}$-centered and $\mathrm{Cu}-$ centered stripes found in many approaches 16, 23, 24, and the small energy barrier between these solutions (Fig. (4). The soft collective mode is a good candidate to mediate pairing between holes and to produce non-Fermi liquid anomalies in the normal state. A related scenario was presented in Ref. [5] but the origin of the softening was not clear.

We use a three-band model for cuprates with the same LDA parameter set 25] as in Ref. [16].

We start by reviewing some mean-field results 5 , 16, 26] in order to illustrate the doping dependent charge and spin textures on which the following computations are based. At very dilute doping $(x \lesssim 0.03)$ due to the long-range Coulomb interaction (not included in our calculations) each hole will be close to an acceptor preventing the formation of stripes. The lowest energy one-hole solution consists of a self-trapped state similar to the Zhang-Rice state[27] as found in HF [5, 26].

As doping increases, the donor potential becomes more uniform and screened, favoring the formation of stripes. Experiment shows that stripes are parallel to the $\mathrm{Cu}$ $\mathrm{O}$ bond except at dopings $0.03 \lesssim x \lesssim 0.05$ where diagonal stripes have been observed [28]. Those may be an intermediate state between the isolated polarons and the vertical stripes and probably also require the longrange Coulomb interaction to be stable. For simplicity we skip this phase and consider vertical metallic solutions. Weak one-dimensional instabilities probably relevant at low temperatures are intentionally suppressed in our clusters due to finite size effects.

In Ref. [16] we have shown that the most favorable lowdoping metallic mean-field stripe is centered on $\mathrm{O}$ sites bridging two vertical $\mathrm{Cu}$ legs, denoted as bond-centered (BC) in one band models 23, 24. Fig. 1 (a) shows a cross section of the charge modulation perpendicular to the stripe. For larger concentrations the $\mathrm{BC}$ solution becomes degenerate with the site centered (SC) one at $x_{0} \approx 0.21[16]$ whose charge profile is shown in Fig. 11(c).

The mean-field bands for a BC stripe lattice as reported in Ref. [16] are shown in Fig. 2. Here we discuss in more detail the symmetry which determines the selection rules for optical transitions. Roughly speaking the flat bands labeled S and P correspond to symmetric (S) and antisymmetric $(\mathrm{P})$ combinations of orbitals centered on the two legs of $\mathrm{Cu}$ that form the core of the stripe (sites 2 and 3 in Fig. 1). The band crossing the chemical potential (hereafter the "active band") is due to the orbitals centered on the core $\mathrm{O}$ leg of the stripe [at 2.5

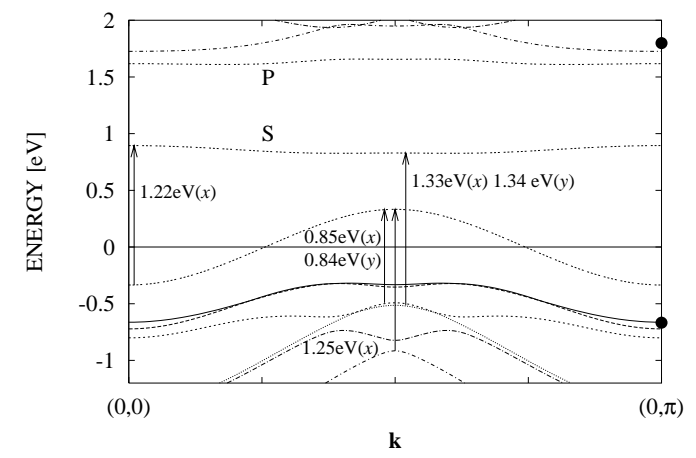

FIG. 2: Electron mean-field bands measured from the chemical potential for the momentum in the direction of the stripe $(d=7, x=0.071)$. The arrows are the lowest energy dipole allowed mean-field transitions in a $d \times 4$ cluster labeled by energy and polarization. Notice that the $x$ polarization is perpendicular to the stripe. We also plot the energies of the AF insulating bands at momentum $\mathbf{k}=(0, \pi)$ (full dots) measured from the same reference energy

in Fig. 1(a)]. The antisymmetric $\mathrm{Cu}$ orbital combination mixes with the core $\mathrm{O}$ orbital pushing upwards (downwards) the $\mathrm{P}$ band (active band) close to the edge of the stoichiometric bands (marked by the full dots). The lower bands are of mainly $\mathrm{O}$ character. All optical transitions in the $x$ direction indicated in Fig. 2 are of course between even and odd states with respect to the stripe central axis.

Dynamical properties are computed within a real space implementation of the GA+RPA method 22. In this context it is essential to use GA+RPA rather than conventional $\mathrm{HF}+\mathrm{RPA}$ theory due to the above-mentioned fact that the starting point mean-field solution matches the experimentally observed doping-induced incommensurability. As a bonus GA+RPA has been shown to be more accurate than $\mathrm{HF}+\mathrm{RPA}[22]$. Note that stripes in LSCO show a dynamic character on a scale of a few $\mathrm{meV}$ 12] which is not capture by our starting-point meanfield ground state. We expect this to affect the spectra at very low energies as discussed in Ref. [A but not on the scale of the transitions reported Fig. 3 .

Fig. 3 reports the optical conductivity within GA+RPA for various dopings. The RPA optical conductivity for the single-hole solution appropriate at low doping, as discussed above, reproduces the results of HF+RPA[5]: Formation of a doping induced MIR band close to $0.5 \mathrm{eV}$ and doping induced transfer of spectral weight from the charge transfer band to the MIR region in agreement with experiment in this doping range 1].

For distant stripes $(d=7)$ the single-hole MIR band now splits into two bands. The one at higher energy is a band of incoherent particle-hole excitations close to $1.3 \mathrm{eV}$ which provides a theoretical explanation for the HEMIR. The position of this band is nearly indepen- 


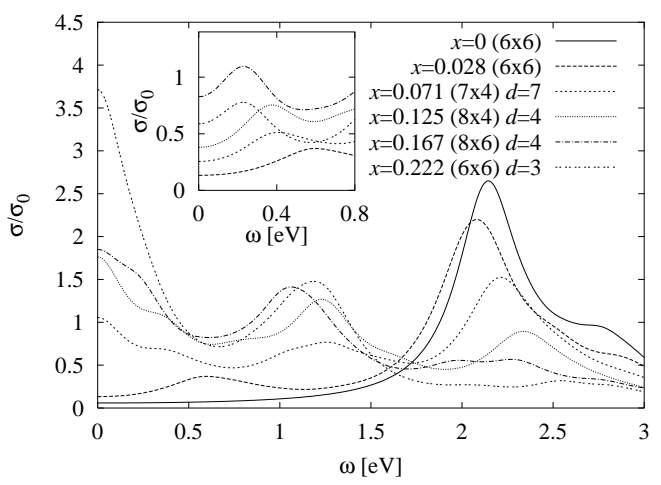

FIG. 3: Optical conductivity labeled by doping, system size and, in the case of stripes, interstripe distance. The units of conductivity are given by $\sigma_{0}=3.6 \times 10^{2}(\Omega \mathrm{cm})^{-1}$ with a background dielectric constant $\epsilon_{b}=2.6$ (see Ref. [5 and caption of Fig. 1). The curve labeled $x=0.028$ corresponds to the single-hole solution. For larger dopings the figure is an average over the electric field directions parallel and perpendicular to the BC stripes. The inset shows the low energy spectra excluding the Drude component. We used a Lorentzian broadening of $0.2 \mathrm{eV}$.

dent of doping and can be understood in terms of transitions within the stripe band structure at mean-field level shown in Fig. 2. Indeed the HEMIR is mainly formed by the $1.33 \mathrm{eV}(x)$ and $1.34 \mathrm{eV}(y)$ mean field transitions with similar oscillation strengths. This negligible renormalization of the mean field transitions by the RPA is characteristic for incoherent particle-hole excitations. The other MIR band, shown also in the inset of Fig. 3 , is a low-energy collective mode and has no mean-field counterpart. In fact the $0.85 \mathrm{eV}(x)$ transition which has a strong oscillator strength in mean-field does not show up in RPA and instead the low-energy collective mode appears which is also polarized perpendicular to the stripes. A similar mode was found in a study of stripes within the $t-t^{\prime}-t^{\prime \prime}-J$ model 29]. The other transitions reported in Fig. 2 have much smaller spectral weights.

To characterize the LEMIR band we compute the so called transition charges $n_{i}^{\mu} \equiv\left\langle 0\left|\hat{n}_{i}\right| \mu\right\rangle$ where $\mu$ labels RPA excitations. $n_{i}^{\nu}$ is proportional to the timedependent charge fluctuation $\delta n_{i}$ that would occur at frequency $\omega_{\nu}$ if the state $\nu$ were weakly excited[30.

In Fig. 1 (b) we show the charge fluctuation associated with the LEMIR mode. It is very similar to the difference in charge density between the BC and SC stripe, indicating that it corresponds to lateral displacements of the stripe. In fact, if we approximate the charge modulation by $\cos \left(q_{\mathrm{cdw}} r+\theta\right)$ with $q_{\mathrm{cdw}}=2 \pi(2 \epsilon, 0)$, this oscillation can be interpreted as a time-dependent fluctuation of the phase $\theta$ and thus the LEMIR excitation can be identified with a phason. Optically active phasons have zero momentum, but naturally a band of phasons exist with a well-defined dispersion relation. In continuum

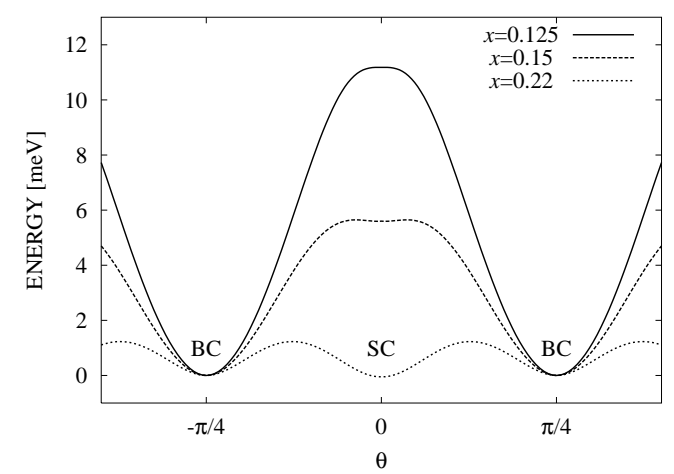

FIG. 4: Mean field energy per stripe cell (8 Cu's) as a function of the collective phase $\theta$ of the CDW for various doping levels and for $d=4$ stripes in a $4 d \times 12$ system.

models phasons are massless Goldstone modes whereas here the commensurability of $q_{\mathrm{cdw}}$ with the lattice makes them have a finite energy at zero momentum. This energy however is small and decreases as doping increases. Since the $\mathrm{BC}$ and $\mathrm{SC}$ state become quasidegenerate at $x_{0}$ it is natural to expect that the phason softening is related to the quasidegeneracy between these states.

In order to substantiate this idea we compute the energy landscape for "intermediate" solutions constrained to be stripes centered between $\mathrm{Cu}$ and $\mathrm{O}$. For each intermediate state we perform a Fourier analysis and extract the phase $\theta$ of the first Fourier component of the charge-density-wave (CDW) modulation. Fig. A shows the energy for $d=4$ stripes and different dopings. This provides an upper bound for the energy along the path connecting $\mathrm{BC}(\theta=\pi / 4)$ and $\mathrm{SC}(\theta=0)$ solutions where $\theta$ plays the role of a collective coordinate. The curves are periodic in $\theta$ with period $2 \pi / d$ corresponding to a translation by one elementary unit cell. Remarkably the curve acquires an extra periodicity close to optimum doping corresponding to the previously found quasidegeneracy between SC and $\mathrm{BC}$ solutions $16,23,24$.

RPA is essentially an harmonic approximation of the energy landscape around the mean field solution (SC or $\mathrm{BC}$ ). The energy squared of each RPA mode is proportional to the curvature (or "stiffness") of the corresponding parabolic energy approximation when the system is displaced from the stationary state in the direction of the mode eigenvector (in our case parametrized by the collective coordinate $\theta$ ). Fig. 1 shows that as doping increases the stiffness decreases showing explicitly that the softening of the LEMIR feature is due to the quasidegeneracy between $\mathrm{BC}$ and $\mathrm{SC}$ stripes. Of course the problem is very anharmonic close to $x_{0}$ and RPA provides only a rough estimate to the phason energy. Moreover, since the barrier is strongly reduced close to $x_{0}$ we expect that anharmonic corrections will make the phason even softer and the "true" ground state will be a fluctuating mixture of $\mathrm{BC}$ and $\mathrm{SC}$ solutions. 


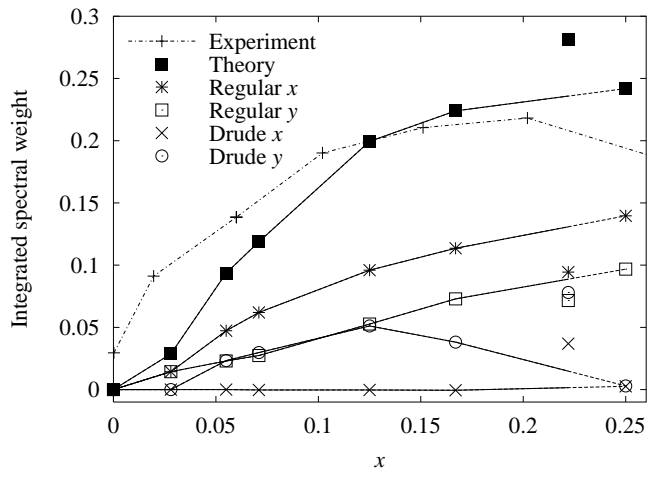

FIG. 5: Experimental[ [n and theoretical optical conductivity spectral weight integrated up to $1.5 \mathrm{eV}$ vs. $x$. The spectral weight is converted to an effective number of electrons $N_{\text {eff }}$ as in Ref. 11. The background dielectric constant $\epsilon_{b}$ is our only free parameter and was adjusted to make the theoretical and experimental intensities to have an overall agreement. We also show the computed Drude and regular contributions in each direction. The dashed lines indicate the region of metastability of the $d=4$ solution. The points not joined by lines correspond to the $d=3$ solution which becomes more stable in that region.

In Fig. 5 we show the optical conductivity spectral weight integrated up to an energy of $1.5 \mathrm{eV}\left[N_{\text {eff }}(1.5 \mathrm{eV})\right]$ in comparison with experimental data from Ref. [1]. The lower curves report the regular $(\omega>0)$ and Drude $(\omega=0)$ contributions to $N_{\text {eff }}(1.5 \mathrm{eV})$ in each direction.

For $x<1 / 8$ the number of stripes, which act as low-energy light absorbers, increases linearly with doping keeping its electronic structure practically unchanged [16. Accordingly $N_{\text {eff }}(1.5 \mathrm{eV})$ increases roughly linearly with doping. For $1 / 8>x>x_{1}$ $\left(x_{1} \sim 0.22-0.23\right)$ the number of absorbers (stripes) get locked and the evolution of the spectral weight is related to changes in the electronic structure of each stripe. Especially the shift of the chemical potential from the center of the active band [16] leads to a depression of Drude weight in the stripe direction (joined circles) and results in a slower increase of spectral weight with doping which correlates well with experiment.

For $x>x_{1} d=3$ stripes become the mean-field ground state. These solutions tend to have a larger spectral weight due to the strong Drude weight in both directions (unjoined points in Fig. 5). The nature of the ground state however is not clear in this overdoped regime and therefore our results become more qualitative than quantitative. Indeed $d=3$ stripes have not been observed in LSCO and theoretically a combination of different solutions would likely be more appropriate 16] which is beyond the scope of this work.

To conclude we have computed the optical conductivity of metallic stripes within a GA+RPA approach. Striped domain walls induce two MIR excitations: At around $1.3 \mathrm{eV}$ a HEMIR band appears which is related to interband transitions within the stripe band structure. Further on we found a collective mode (i.e. the LEMIR band) which softens as a function of doping due to the suppression of the energy barrier between quasidegenerate BC and SC stripe solutions. These features are in good agreement with experiments for positions and relative intensities with parameters fixed by first principle computations as in Ref. [16. It is worth speculating that scattering of holes moving along and perpendicular to the stripes with the soft collective mode may be responsible for both the anomalous normal state behavior and the superconducting pairing in cuprates.

After this work was completed Ref. [31] was posted in which by studding temperature effects, MIR features in isostructural $\mathrm{La}_{2} \mathrm{NiO}_{4.133}$ were also linked to stripes.

J.L. acknowledges hospitality at ICTP during this work and G.S. acknowledges support from the Deutsche Forschungsgemeinschaft.

[1] S. Uchida et al., Phys. Rev. B 43, 7942 (1991).

[2] M. Suzuki, Phys. Rev. B 39, 2312 (1989).

[3] T. Timusk et al, Phys. Rev. B 38, 6683 (1988); C. Varma et al., Phys. Rev. Lett. 63, 1996 (1989); A. Virosztek and J. Ruvalds, Phys. Rev. B 42, 4064 (1990);

[4] S. Caprara et al., Phys. Rev. Lett. 88, 147001 (2002).

[5] J. Lorenzana and L. Yu, Phys. Rev. Lett. 70, 861 (1993).

[6] M. Terauchi and M. Tanaka, Micron 30, 371 (1999).

[7] J. M. Ginder et al., Phys. Rev. B 37, 7506 (1988).

[8] J.M. Leng et al., Phys. Rev. B 43, 10582 (1991).

[9] J. M. Tranquada et al., Nature (London) 375, 561 (1995).

[10] J. M. Tranquada et al., Phys. Rev. B 54, 7489 (1996).

[11] J. M. Tranquada et al., Phys. Rev. Lett. 78, 338 (1997).

[12] K. Yamada et al., Phys. Rev. B 57, 6165 (1998).

[13] M. Arai et al., Phys. Rev. Lett. 83, 608 (1999).

[14] M. Arai et al., Int. J. Mod. Phys. B 14, 3312 (2000).

[15] J. Zaanen and O. Gunnarsson, Phys. Rev. B 40, 7391 (1989); D. Poilblanc and T. M. Rice, Phys. Rev. B 39, 9749 (1989); K. Machida, Physica C, 158, 192 (1989), H. J. Schulz, Phys. Rev. Lett. 64, 1445 (1990).

[16] J. Lorenzana and G. Seibold, Phys. Rev. Lett. 89, 136401 (2002).

[17] M. C. Gutzwiller, Phys. Rev. 137, A1726 (1965).

[18] A. Ino et al., Phys. Rev. Lett. 79, 2101 (1997).

[19] N. Harima et al., Phys. Rev. B 64, R220507 (2001).

[20] T. Noda, H. Eisaki, and S. Uchida, Science 286, 265 (1999).

[21] Y. Wang and N. P. Ong, Proc. Natl. Acad. Sci. U.S.A. 98, 11091 (2001).

[22] G. Seibold and J. Lorenzana, Phys. Rev. Lett. 86, 2605 (2001).

[23] S. R. White and D. J. Scalapino, Phys. Rev. Lett. 80, 1272 (1989).

[24] M. Fleck et al., Phys. Rev. Lett. 84, 4962 (2000).

[25] A. K. McMahan, J. F. Annett, and R. M. Martin, Phys. Rev. B 42, 6268 (1990).

[26] K. Yonemitsu, A. R. Bishop, and J. Lorenzana, Phys. Rev. Lett. 69, 965 (1992).

[27] F. C. Zhang and T. M. Rice, Phys. Rev. B 37, 3759 
(1988).

[28] M. Matsuda et al., Phys. Rev. B 62, 9148 (2000).

[29] T. Tohyama et al., Phys. Rev. Lett. 82, 4910 (1999).

[30] P. Ring and P. Schuck, The nuclear many-body problem
(Springer-Verlag, New York, 1980).

[31] C.C. Homes et al. cond-mat/0207003. 